\title{
Inflammatory Biomarkers for Children with Sleep Disorders: A Review
}

\author{
Nagashree Prabhu ${ }^{1}$ Vabitha Shetty ${ }^{1}$ (1) \\ ${ }^{1}$ Department of Pediatric and Preventive Dentistry, AB Shetty \\ Memorial Institute of Dental Sciences, Nitte (Deemed to be \\ University), Mangalore, Karnataka, India \\ J Health Allied Sci ${ }^{\mathrm{NU}}$ 2022;12:356-360.
}

\begin{abstract}
Address for correspondence Vabitha Shetty, MDS, Department of Pediatric and Preventive Dentistry, AB Shetty Memorial Institute of Dental Sciences, Nitte (Deemed to be University), Mangalore 575018, Karnataka, India (e-mail: docvabitha29@gmail.com).
\end{abstract}

\begin{abstract}
Introduction Obstructive sleep apnea (OSA) syndrome is a highly prevalent problem in children. Unfortunately sleep disorders in children remain largely unreported and underdiagnosed due to various reasons. Overnight in-laboratory polysomnography (PSG) is the gold standard diagnostic method for OSA; however, it has several drawbacks. Hence there is a need for simple alternative diagnostic methods, such as biomarkers. Children with OSA seem to have an inflammatory aspect to its pathophysiology; thus the rationale for evaluating inflammatory biomarkers.

Objective This narrative review aimed to evaluate the existing knowledge regarding inflammatory biomarkers for children with sleep disorders.

Materials and Methods An extensive search of all literature linked to the topic was performed in PubMed, Medline, EBSCOhost, Cochrane library database CENTRAL, and Google Scholar search engine. Keywords used in the search included combinations of the following: inflammation, sleep disorders, children, and biomarkers. Studies in the list of references of relevant literature were also included. A total of 155 articles published in the period from 1997 to 2021 were initially included in this review. Selected articles comprised original research, meta-analysis, and systematic reviews. Results Studies have mainly centered their investigations on serum inflammatory biomarkers, namely interleukin-6, tumor necrosis factor- $\alpha$, and C-reactive protein. Very few studies investigated salivary inflammatory biomarkers in children.

Keywords

- inflammation

- sleep disorders

- children

- biomarkers

Conclusion Evidence suggests that children with sleep disorders have elevated levels of salivary as well as serum inflammatory biomarkers in comparison to normal healthy children. Also increased levels of these biomarkers correlate with disease severity. However, more studies are needed to determine the sensitivity and specificity of salivary inflammatory biomarkers for children with sleep disorders.
\end{abstract}

\section{Introduction}

Obstructive sleep apnea syndrome (OSAS) is a common sleep disorder in children affecting 0.7 to $10.3 \%$ of the population. ${ }^{1,2}$ It is mostly linked to morphological features such a long, narrow face, nasal breathing difficulty (allergies, recurrent colds), and mouth breathing. The dentist's involvement in the early diagnosis of anatomic risk factors or symptoms has grown increasingly to be essential in recent years. $^{3}$ received

August 27, 2021

accepted

DOI https://doi.org/

10.1055/s-0042-1742371.

ISSN 2582-4287. 
OSAS can occur in children of all ages, from neonates to adolescents, and has significant medical, psychological, and social consequences, which can hamper the quality of life and can be potentially fatal. ${ }^{4}$ Due to the far-reaching consequences that sleep problems could have on the growing child, it is imperative that focus should be on early diagnosis and treatment. Unfortunately sleep problems in children remain largely underdiagnosed, which could be due to a lack of physician's awareness or patients reluctance to undergo screening or unattainable diagnostic/screening tools. ${ }^{5}$

The gold standard diagnostic approach for obstructive sleep apnea (OSA) at any age is an overnight in-laboratory polysomnography (PSG) assessment. ${ }^{6}$ Unfortunately, overnight PSGs are time-consuming, may cause significant inconvenience to the child and caregivers, and are not universally available. Alternative diagnostic procedures have become increasingly important in recent years. ${ }^{7}$ Simple methods including survey, pulse oximetry, audiotaping, videotaping, abbreviated PSG, or multichannel recordings have all been evaluated, with varying degrees of success. ${ }^{8-10}$ Among several other diagnostic methods, biomarker identification has recently gotten a lot of attention.

A biomarker is a component that may be quantified and analyzed objectively as an indication of normal biologic events, pathogenic processes, or the pharmacologic reaction to a therapeutic treatment. ${ }^{11}$ If accurate and reliable biomarkers for OSAS-related morbidities can be developed and validated, prompt risk stratification and tailored treatment interventions with improved outcomes can be implemented. ${ }^{12}$

Recent attention has focused on various inflammatory markers for sleep disorders, which could facilitate diagnosis; however, an ideal marker has yet to be discovered. Most studies have centered on the assessment of serum inflammatory biomarkers, namely interleukin (IL)-6, tumor necrosis factor$\alpha(\mathrm{TNF}-\alpha)$, and C-reactive protein (CRP) levels in adult population with sleep disorders. A recent scoping review by Ibáñez-Del Valle et al have assessed changes in inflammatory markers in saliva and their association with sleep quality measurements in healthy subjects and in sleep-related disorders. However, their review found only two studies related to children. ${ }^{13}$ Therefore, there is a lacuna of knowledge regarding inflammatory biomarkers for the evaluation of sleep disorders in children. In the present review, we sought to examine the existing literature regarding inflammatory biomarkers among children with sleep disorders.

\section{Aim}

The aim of this narrative review was to evaluate the existing literature regarding inflammatory biomarkers among children with sleep disorders.

\section{Search Methodology}

An extensive search of all literature linked to the topic was performed in PubMed, Medline, EBSCOhost, Cochrane library database CENTRAL, and Google Scholar search engine. Keywords used in the search included combinations of the following: inflammation, sleep disorders, children, and biomarkers. Studies in the list of references of relevant literature were also included. A total of 155 articles published in the period from 1997 to 2021 were initially included in this review. Selected articles comprised original research, metaanalysis, and systematic reviews. Duplicate articles, irrelevant articles, non-English articles, unpublished data, and case reports were discarded. After reviewing the full text of the selected articles, we have chosen 14 articles for this review.

\section{Role of Inflammatory Biomarkers in Sleep Disorders}

Even though the precise connections between OSA and the inflammatory cascade are unknown, it appears to have an inflammatory component. Snoring causes tissue injury in the upper airway, demonstrated experimentally to induce higher levels of biomarkers such as IL-8. ${ }^{12}$

Repetitive hypoxia and reoxygenation associated with OSA are believed to cause oxidative stress ${ }^{13,14}$ and the production of reactive oxygen species (ROS), which may play a role in the activation of an inflammatory response in OSA patients. ${ }^{15}$

ROS could be more than just a harmful consequence of metabolism; it could also be a tightly regulated molecule with essential signaling qualities that can activate inflammatory pathways. ${ }^{16}$ They trigger several proinflammatory cytokines, such as TNF- $\alpha$, IL- 6 , and IL-8, via the transcription factors like nuclear factor-kB (NF-kB), and activator protein $1 .{ }^{17} \mathrm{CRP}$ is produced in reaction to proinflammatory cytokines, most notably IL-6, but also IL- 1 and TNF- $\alpha .^{18}$

The proinflammatory transcription factor NF-KB is thought to be the master switch that regulates expression of several genes taking part in inflammation, metabolic syndrome, and atherosclerosis. ${ }^{19}$ Repeated hypoxia and reoxygenation selectively trigger the proinflammatory transcription factor NF-KB, and individuals with OSA have been found to have elevated NF-KB levels in neutrophils and monocytes ${ }^{20}$ that correspond with OSA severity and are lowered with continuous positive airway pressure treatment. ${ }^{21}$

\section{Inflammatory Biomarkers in Diagnosis of Sleep Disorders 22,23}

\author{
1. CRP \\ 2. TNF- $\alpha$ \\ 3. IL-6, IL-8, and IL-1
}

\section{C-Reactive Protein}

Tillet and Francis discovered CRP in 1930, which is a homopentameric acute-phase inflammatory protein with a high degree of conservation in plasma. ${ }^{24}$ It is largely produced by liver hepatocytes, although it has also been found in smooth muscle cells, macrophages, endothelial cells, lymphocytes, and adipocytes. ${ }^{25}$ Its expression is upregulated during inflammatory disorders such as rheumatoid arthritis, 
certain cardiovascular diseases, and infection. ${ }^{26}$ As an acutephase protein, CRP plasma levels vary by at least $25 \%$ during inflammatory conditions. ${ }^{27}$ Within 24 to 72 hours of significant tissue injury, CRP plasma levels rise from roughly $1 \mathrm{~g} / \mathrm{mL}$ to over $500 \mathrm{~g} / \mathrm{mL}^{28}$ Proinflammatory cytokines, particularly IL-6, but also IL- 1 and TNF- $\alpha$, stimulate CRP production. ${ }^{18}$

In adults, the evidence suggests that there is a substantial link between OSAS and high-sensitivity CRP levels that is independent of other well-known risk factors. ${ }^{29}$ However, the relationship between OSAS in children and CRP has been the subject of various studies with contradictory results. Tauman et al examined 81 children, who were subjected to a polysomnographic examination for OSAS. In a fasting state, CRP levels and lipid profiles were determined the next morning. Significant relationships appeared between log CRP levels and apnea-hypopnea index, arousal index, and the lowest nocturnal arterial oxygen saturation, which remained significant after body mass index was taken into account. Furthermore, in comparison to $62 \%$ of children with normal CRP levels, 94\% of the children with high log CRP levels showed excessive daytime sleep deprivation and/or learning difficulties. ${ }^{30}$ Larkin et al found similar results in an adolescent sample as well. ${ }^{31}$

Kheirandish-Gozal et al studied 20 nonobese children with OSAS and discovered significant reductions in CRP levels after OSAS was effectively resolved, demonstrating that OSAS causes CRP elevations in children independent of obesity. Moreover, when they investigated IL-6 and CRP levels in connection to sleep parameters in the context of pediatric OSAS, comparable relationships were found among nonobese children, thereby validating the scientific evidence of this association. $^{32}$ CRP levels were elevated in both nonobese untreated OSAS $(n=25)$ and obese untreated OSAS $(n=37)$ children in a following study by Gozal et al, which aimed to evaluate the metabolic ramifications of OSAS in children. Additionally, CRP levels lowered after intervention in both nonobese $(4.0 \pm 0.9$ to $1.1 \pm 0.2 \mu \mathrm{g} / \mathrm{mL} ; p<0.0001)$ and in obese children $(6.1 \pm 1.0$ to $2.4 \pm 0.6 \mu \mathrm{g} / \mathrm{mL} ; p<0.001) .{ }^{33}$

Li et al measured CRP levels in children with OSA and evaluated the impact of OSA treatment on CRP levels in the blood. OSA was observed in 45 of 141 children, and those with moderate disease [Obstructive apnoea index $(\mathrm{OAI})>5$ ] had considerably higher CRP levels than their non-OSA counterparts. Sixteen children were treated, and their serum CRP levels dropped significantly as a result of the intervention. Following treatment for OSA, there was a substantial association between changes in CRP and changes in OAI. ${ }^{34}$ Similar results were reported in a meta-analysis by Ingram and Matthews and Bhattacharjee et al. ${ }^{35,36}$

There is evidence suggestive of elevated CRP levels in childhood OSAS. Notably, CRP may serve as a valuable biomarker for the diagnosis of OSAS in children.

\section{Tumor Necrosis Factor- $\alpha$}

TNF- $\alpha$ was identified in 1975 , later isolated and characterized in $1984 .{ }^{37}$ Initially identified as a serum factor that triggers cell death in tumor cells and thought at that time to be a promising target as a cancer treatment, TNF was eventually recognized to be a viable target for the therapy of inflammatory diseases. ${ }^{38,39}$

Mononuclear macrophages, natural killer cells, and other immune cells secrete TNF- $\alpha .{ }^{40}$ It is important for host defense and has a role in the etiology of a variety of medical conditions, including malignancies, infectious diseases, autoimmune diseases, cardiovascular diseases, and atherosclerosis. ${ }^{40}$ TNF- $\alpha$ has been linked to sleep modulation by activating NF-кB pathways, which leads to increased expression of the adenosine A1 receptor, cyclooxygenase- 2 , and NO synthase, all of which are assumed to be important in sleep regulation. ${ }^{17,41}$

Vgontzas et al assessed plasma cytokines levels in disorders of excessive daytime sleepiness in adult population. Blood was drawn to measure plasma TNF- $\alpha$, IL-1 $\beta$, and IL-6 using enzyme-linked immunosorbent assay. Patients with OSA had plasma TNF- $\alpha$ concentration significantly elevated compared with that of control group. ${ }^{42}$ Similar results were reported by Gozal et al and Khalyfa et al. ${ }^{43,44}$

Li et al evaluated serum cytokine levels in children with and without OSA and explored the effects of OSA treatment, that is, adenotonsillectomy, on cytokines. ${ }^{35}$ There was no significant difference in TNF- $\alpha$ between the non-OSA and OSA groups. Adenotonsillectomy resulted in a considerable reduction in serum cytokines in children. ${ }^{28}$ Driessen et al assessed for OSAS in 11 adults and 37 children with syndromic craniosynostosis and Treacher Collins syndrome. Malondialdehyde, TNF- $\alpha$, IL-6, and high-sensitivity CRP underwent laboratory analyses. In 23 of the 48 individuals, OSAS was diagnosed. Only TNF- $\alpha$ was found to be considerably greater in both OSAS and non-OSAS patients. $^{45}$

Alexopoulos et al correlated the severity of OSA and morning TNF- $\alpha$ plasma levels in children who underwent PSG. Morning plasma TNF- $\alpha$ levels were similar in mild OSAS, moderate-severe OSAS, and control groups. ${ }^{46}$

Ibáñez-del Valle et al analyzed the alterations in salivary inflammatory biomarkers and their relationships with evaluation of sleep quality parameters. ${ }^{13}$ Eleven articles were pertaining to adults while only two focused on children/ youth population. They primarily assessed the link between sleep and IL-1, IL-6, TNF- $\alpha$, and some other inflammatory markers such as myeloperoxidase (MPO) and prostaglandinendoperoxide synthase 2 . Due to the contradictory results observed with TNF- $\alpha$-levels increased, decreased, and remained unchanged-no general conclusion could be drawn about this cytokine in saliva. ${ }^{47}$

\section{Interleukins}

Interleukins are a type of cytokine that was once considered to be produced only by leukocytes but has now been discovered to be produced by a variety of different bodily cells. They are important in immune cell activation and differentiation, as well as proliferation, maturation, migration, and adhesion. They have anti-inflammatory and proinflammatory effects as well. Their major function is to control growth, differentiation, and activation during inflammatory and immunological reactions. They are a large group of proteins that bind to high-affinity 
receptors on cell surfaces to activate a variety of responses in cells and tissues. They work in both paracrine and autocrine manner. ${ }^{48}$

Interleukins are also used in studies to investigate presence and severity of sleep disorders. This chemokine's expression and production have been shown to be substantially affected by hypoxia. ${ }^{49} \mathrm{Li}$ et al looked for the levels of serum cytokines in children with and without OSA and the impact of OSA treatment on cytokines. ${ }^{35}$ When compared with their non-OSA counterparts, children with OSA had greater serum IL-6 and IL-8 values. Adenotonsillectomy resulted in a considerable decrease in serum cytokines in children. ${ }^{34}$ Driessen et al found that IL-6 levels were not higher in patients with OSAS. ${ }^{45}$

LaVoy et al explored connections between objective measures of sleep and salivary biomarkers in 55 children and adolescents. $\alpha$-Amylase, cortisol, IL- 6 , and IL- 1 were all measured in the first waking saliva samples. Elevated waking IL-1 $\beta$ and IL-6 levels were linked to superior-quality sleep the night before. ${ }^{50}$

\section{Conclusion}

Evidence suggests that children with sleep disorders have elevated levels of salivary as well as serum inflammatory biomarkers in comparison to normal healthy children. Also increased levels of these biomarkers correlate with disease severity. However, more studies are needed to determine the sensitivity and specificity of salivary inflammatory biomarkers for children with sleep disorders. There is insufficient data on the role of biomarkers for OSA in the pediatric population. Future research should be directed toward standardization of inflammatory biomarkers and determination of the precise link to presence and severity of OSA.

\section{Author's Contributions}

V. Shetty has been conducting research in the field of sleep disorders in children since past 5 years. She has two published studies and one under review in this area of research. She has played a significant role in review of literature and article writing. N. Prabhu has played an active role in data collection and article writing.

\section{Conflict of Interest}

None.

\section{References}

1 Gislason T, Benediktsdóttir B. Snoring, apneic episodes, and nocturnal hypoxemia among children 6 months to 6 years old. An epidemiologic study of lower limit of prevalence. Chest 1995; 107(04):963-966

2 Redline S, Tishler PV, Schluchter M, Aylor J, Clark K, Graham G. Risk factors for sleep-disordered breathing in children. Associations with obesity, race, and respiratory problems. Am J Respir Crit Care Med 1999;159(5 Pt 1):1527-1532

3 Huynh NT, Morton PD, Rompré PH, Papadakis A, Remise C. Associations between sleep-disordered breathing symptoms and facial and dental morphometry, assessed with screening examinations. Am J Orthod Dentofacial Orthop 2011;140(06):762-770
4 Dayyat E, Kheirandish-Gozal L, Gozal D. Childhood obstructive sleep apnea: one or two distinct disease entities? Sleep Med Clin 2007;2(03):433-444

5 Owens JA. The practice of pediatric sleep medicine: results of a community survey. Pediatrics 2001;108(03):E51

6 Marcus CL, Brooks LJ, Draper KA, et al; American Academy of Pediatrics. Diagnosis and management of childhood obstructive sleep apnea syndrome. Pediatrics 2012;130(03):e714-e755

7 Gozal D. Serum, urine, and breath-related biomarkers in the diagnosis of obstructive sleep apnea in children: is it for real? Curr Opin Pulm Med 2012;18(06):561-567

8 Lamm C, Mandeli J, Kattan M. Evaluation of home audiotapes as an abbreviated test for obstructive sleep apnea syndrome (OSAS) in children. Pediatr Pulmonol 1999;27(04):267-272

9 Sivan Y, Kornecki A, Schonfeld T. Screening obstructive sleep apnoea syndrome by home videotape recording in children. Eur Respir J 1996;9(10):2127-2131

10 Andreu AL, Chiner E, Sancho-Chust JN, et al. Effect of an ambulatory diagnostic and treatment programme in patients with sleep apnoea. Eur Respir J 2012;39(02):305-312

11 Atkinson Jr AJ, Colburn WA, DeGruttola VG, et al. Biomarkers and surrogate endpoints: preferred definitions and conceptual framework. Clinl pharmacol Ther 2001;69(03):89-95

12 Friberg D. Heavy snorer's disease: a progressive local neuropathy. Acta Otolaryngol 1999;119(08):925-933

13 Ibáñez-Del Valle V, Navarro-Martínez R, Ballestar-Tarín ML, Cauli 0 . Salivary inflammatory molecules as biomarkers of sleep alterations: a scoping review. Diagnostics (Basel) 2021;11(02):278

14 Suzuki YJ, Jain V, Park A-M, Day RM. Oxidative stress and oxidant signaling in obstructive sleep apnea and associated cardiovascular diseases. Free Radic Biol Med 2006;40(10):1683-1692

15 Lavie L. Sleep-disordered breathing and cerebrovascular disease: a mechanistic approach. Neurol Clin 2005;23(04):1059-1075

$16 \mathrm{McCord}$ JM. The evolution of free radicals and oxidative stress. Am J Med 2000;108(08):652-659

17 Haddad JJ. Pharmaco-redox regulation of cytokine-related pathways: from receptor signaling to pharmacogenomics. Free Radic Biol Med 2002;33(07):907-926

18 Zhang D, Sun M, Samols D, Kushner I. STAT3 participates in transcriptional activation of the C-reactive protein gene by interleukin-6. J Biol Chem 1996;271(16):9503-9509

19 Schäfer H, Pauleit D, Sudhop T, Gouni-Berthold I, Ewig S, Berthold HK. Body fat distribution, serum leptin, and cardiovascular risk factors in men with obstructive sleep apnea. Chest 2002;122(03): 829-839

20 Htoo AK, Greenberg H, Tongia S, et al. Activation of nuclear factor kappaB in obstructive sleep apnea: a pathway leading to systemic inflammation. Sleep Breath 2006;10(01):43-50

21 Yamauchi M, Tamaki S, Tomoda K, et al. Evidence for activation of nuclear factor kappaB in obstructive sleep apnea. Sleep Breath 2006;10(04):189-193

22 Archontogeorgis K, Nena E, Papanas N, Steiropoulos P. Biomarkers to improve diagnosis and monitoring of obstructive sleep apnea syndrome: current status and future perspectives. Pulm Med 2014;2014:930535

23 Kheirandish-Gozal L, Gozal D. Sleep Disordered Breathing in Children. New York: Springer; 2012

24 Tillett WS, Francis T. Serological reactions in pneumonia with a non-protein somatic fraction of Pneumococcus. J Exp Med 1930; 52(04):561-571

25 Sproston NR, Ashworth JJ. Role of C-reactive protein at sites of inflammation and infection. Front Immunol 2018;9:754

26 Du Clos TW, Mold C. C-reactive protein: an activator of innate immunity and a modulator of adaptive immunity. Immunol Res 2004;30(03):261-277

27 Gabay C, Kushner I. Acute-phase proteins and other systemic responses to inflammation. N Engl J Med 1999;340(06):448-454 
28 Ciubotaru I, Potempa LA, Wander RC. Production of modified Creactive protein in U937-derived macrophages. Exp Biol Med (Maywood) 2005;230(10):762-770

29 Gozal D, Kheirandish-Gozal L, Bhattacharjee R, Kim J. C-reactive protein and obstructive sleep apnea syndrome in children. Front Biosci (Elite Ed) 2012;4:2410-2422

30 Tauman R, Ivanenko A, O'Brien LM, Gozal D. Plasma C-reactive protein levels among children with sleep-disordered breathing. Pediatrics 2004;113(06):e564-e569

31 Larkin EK, Rosen CL, Kirchner HL, et al. Variation of C-reactive protein levels in adolescents: association with sleep-disordered breathing and sleep duration. Circulation 2005;111(15): 1978-1984

32 Kheirandish-Gozal L, Capdevila OS, Tauman R, Gozal D. Plasma Creactive protein in nonobese children with obstructive sleep apnea before and after adenotonsillectomy. J Clin Sleep Med 2006;2(03):301-304

33 Gozal D, Capdevila OS, Kheirandish-Gozal L. Metabolic alterations and systemic inflammation in obstructive sleep apnea among nonobese and obese prepubertal children. Am J Respir Crit Care Med 2008;177(10):1142-1149

34 Li AM, Chan MH, Yin J, et al. C-reactive protein in children with obstructive sleep apnea and the effects of treatment. Pediatr Pulmonol 2008;43(01):34-40

35 Ingram DG, Matthews CK. Effect of adenotonsillectomy on creactive protein levels in children with obstructive sleep apnea: a meta-analysis. Sleep Med 2013;14(02):172-176

36 Bhattacharjee R, Kheirandish-Gozal L, Kaditis AG, Verhulst SL, Gozal D. C-reactive protein as a potential biomarker of residual obstructive sleep apnea following adenotonsillectomy in children. Sleep (Basel) 2016;39(02):283-291

37 Carswell EA, Old LJ, Kassel RL, Green S, Fiore N, Williamson B. An endotoxin-induced serum factor that causes necrosis of tumors. Proc Natl Acad Sci U S A 1975;72(09):3666-3670

38 Seymour HE, Worsley A, Smith JM, Thomas SH. Anti-TNF agents for rheumatoid arthritis. Br J Clin Pharmacol 2001;51(03): 201-208
39 Ghosh S. Anti-TNF therapy in Crohn's disease. Novartis Found Symp 2004;263:193-205, discussion 205-218

40 Nadeem R, Molnar J, Madbouly EM, et al. Serum inflammatory markers in obstructive sleep apnea: a meta-analysis. J Clin Sleep Med 2013;9(10):1003-1012

41 Kheirandish-Gozal L, Gozal D. Obstructive sleep apnea and inflammation: proof of concept based on two illustrative cytokines. Int J Mol Sci 2019;20(03):459

42 Vgontzas AN, Papanicolaou DA, Bixler EO, Kales A, Tyson K, Chrousos GP. Elevation of plasma cytokines in disorders of excessive daytime sleepiness: role of sleep disturbance and obesity. J Clin Endocrinol Metab 1997;82(05):1313-1316

43 Gozal D, Serpero LD, Kheirandish-Gozal L, Capdevila OS, Khalyfa A, Tauman R. Sleep measures and morning plasma TNF- $\alpha$ levels in children with sleep-disordered breathing. Sleep 2010;33(03): 319-325

44 Khalyfa A, Serpero LD, Kheirandish-Gozal L, Capdevila OS, Gozal D. TNF- $\alpha$ gene polymorphisms and excessive daytime sleepiness in pediatric obstructive sleep apnea. J Pediatr 2011;158(01): $77-82$

45 Driessen C, Plomp RG, van der Spek PJ, et al. Is there an effect of obstructive sleep apnea syndrome on oxidative stress and inflammatory parameters in patients with craniofacial anomalies? J Craniofac Surg 2013;24(06):1908-1913

46 Alexopoulos EI, Theologi V, Malakasioti G, et al. Obstructive sleep apnea, excessive daytime sleepiness, and morning plasma TNF- $\alpha$ levels in Greek children. Sleep (Basel) 2013;36(11):1633-1638

47 Tanaka T, Narazaki M, Kishimoto T. IL-6 in inflammation, immunity, and disease. Cold Spring Harb Perspect Biol 2014;6(10): a016295

48 Hirani N, Antonicelli F, Strieter RM, et al. The regulation of interleukin- 8 by hypoxia in human macrophages-a potential role in the pathogenesis of the acute respiratory distress syndrome (ARDS). Mol Med 2001;7(10):685-697

49 LaVoy EC, Palmer CA, So C, Alfano CA. Bidirectional relationships between sleep and biomarkers of stress and immunity in youth. Int J Psychophysiol 2020;158:331-339 